\title{
Adherence to colorectal cancer screening
} Salem Youssef Mohamed ${ }^{\mathbf{1}}$, Hend Samir $\mathbf{A l i}^{2}$ *, Ayman Fathy Elsayied, ${ }^{I}$ Internal Medicine Department, Faculty of Medicine, Zagazig University, Egypt ${ }^{2}$ Internal Medicine Department, Belbeis Central Hospital, M.B.B.CH Zagazig University, Egypt

\section{Corresponding author:}

Hend Samir Ali

A resident of Internal Medicine at Belbeis Central Hospital, M.B.B.CH Zagazig University

Email address: drhendabozeid@ gmail.com.

Sharkia, Zagazig,

Cell phone: +201285042522

Conflict of interest: The authors declare no conflict of interest.

Funding sources: The authors have no funding to report.

Date of submission: November 15, 2021.

Revised: December 18, 2021.

Accepted: January 11, 2022.

First online: January 13, 2022.

\section{Abstract:}

Background: Colorectal cancer is the third most common cancer in the world. Randomized trials have demonstrated the efficacy of screening. In this review, we discussed the benefits of colonoscopy \&other screening tests and their role in decreasing the mortality rate from CRC. Aim of work: this minireview discusses the different CRC screening modalities and the adherence of populations to these procedures.

Keywords: Colorectal cancer, screening, adherence, guidelines.

\section{Introduction:}

Colorectal cancer (CRC) is one of the most common cancers worldwide. Between one and two million new cases are diagnosed every year, thus making CRC the third most common cancer and the fourth most common cause of cancer-related death, with 700,000 deaths per year. By gender, $\mathrm{CRC}$ is the second most common cancer in women $(9.2 \%)$ and the third in men (10\%) [1].

Colorectal cancer (CRC) is increasing among young individuals in the Arab world, Egypt, and other world regions [2].

In Egypt, no accurate formal prevalence rates; however, early reports showed that 
colorectal cancer is the 7th commonest cancer among Egyptians. From the practice point of view, colon cancer is prevalent among different age groups of Egyptians, and no age seems immune against the development of this cancer. [3].

In the past decade, CRC incidence has been observed worldwide. Additionally, there is an increase in the prevalence of $\mathrm{CRC}$ in the younger population, and new cases are expected to increase among the younger people aged 20-49 years by 2030[3].

Although more than $90 \%$ of CRC cases are diagnosed in individuals over age 55, CRC incidence rises in younger populations. For example, Egypt, Saudi Arabia, the Philippines, and Iran have CRC incidence rates in individuals under age 40 of $38 \%, 21 \%, 17 \%$, and $15 \%-35 \%$, respectively. This is compared with only $2 \%-8 \%$ of new cases in the U.S[4].

Despite the availability of screening modalities, many populations refused to undergo screening, as they are doing well and there is no complaint. They refuse to be screened, especially with invasive procedures such as colonoscopy. [5].

\section{Risk Factors for Colorectal Cancer:}

Factors that increase the risk of developing colorectal cancer can be divided into two categories: modifiable factors and non-modifiable factors. Non-modifiable risk factors include having inflammatory bowel disease, some hereditary factors, and old age [6].

Modifiable factors include obesity, lack of physical activity, a diet high in red and processed meat, smoking, and excessive drinking of alcohol and are all known to be associated with the occurrence of colorectal cancer [6].

\section{Age:}

More than $90 \%$ of the patients diagnosed with colorectal cancer were found to be more than 55 years old in a study conducted by Li and colleagues. Age at diagnosis also affects survival. Other studies report a poor outcome in younger colorectal cancer patients despite previous findings that advanced associate age with poor survival.[7].

Generally, colorectal cancer in young patients usually presents with poor tumor morphology and late-stage diagnosis than older individuals and other factors that lead to poor survival [7]. A large population-based study reported that the most senior group of patients (> 80 years) have almost twice the risk of death after the operation with a hazard ratio of $1.95,95 \%$, after controlling for other factors [7]. 
This finding is explained by a higher percentage of comorbidities and complications experienced by older patients after surgery. This is agreed by Stornes and colleagues, who claimed that age influences the selection of treatment modalities in older patients; for example, older patients may be treated less often with surgery (including major radical surgery) or radiochemotherapy in more local recurrence and poorer eventual outcomes. This suggests a more complex causal pathway [8].so it is important to be adherent to the international guidelines regarding the age of the populations, according to the latest report of the American colorectal cancer guidelines [9]. Relatives of patients with colorectal cancer may begin their screening at an earlier age [9].

\section{Sex:}

The incidence of colorectal cancer is considerably higher in men than women in many countries worldwide. Findings on the influence of sex differences in colorectal cancer prognosis have been less consistent. Some studies reported better survival in women, while others did not find any significant difference [10].

An analysis of a large representative cohort comprising 24 clinical trials that enrolled more than 30,000 colon cancer patients between 1978 and 2003 found that women with earlystage colon cancer have significantly higher survival rates than men [11].

\section{Race:}

Different races or ethnic groups may have different cultures, lifestyles, environments, and food preferences, and these factors may influence their health outcomes in general. Racial disparities have been reported to affect the survival of colorectal cancer in many countries [12].

\section{Smoking}

Investigators carried out a study on the relationship between smoking and the survival of 10,794 rectal cancer cases in Ireland. Current smokers with rectal cancer were significantly poorer survival $(\mathrm{HR}=1.15,95 \% \mathrm{CI}$ 1.06-1.24) than non-smokers. Still, the association was insignificant in ex-smokers diagnosed with rectal cancer [13].

\section{Diabetes}


There is ample evidence of the association of four metabolic syndrome disease characteristics (diabetes, obesity, hypertension, and dyslipidemia) to progression-free survival in Stages I to Stages III colorectal cancer [14].

Diabetes mellitus had a significant positive association with progression-free survival. They found that the use of Metformin in the treatment of diabetic patients improved their outcome of progression-free survival [14].

On the other hand, an extensive systematic review and meta-analysis involving 21 eligible cohorts, which involved more than one million patients of colorectal cancer, had different findings. This meta-analysis indicates that colorectal cancer patients with pre-existing diabetes mellitus were adversely associated with lower (worse) overall survival. However, the association was not significant with cancer-specific survival [15].

\section{Site}

The prognosis of colorectal cancer has been shown to vary depending on the anatomical site of the tumor. Lee and colleagues reported that patients with cancer at the rectum had better survival than those at the colon [16].

\section{Stage:}

As the stage of disease at diagnosis advances, survival becomes considerably shorter. Aravani and colleagues reported that $93 \%$ of patients who presented with earlier-stage disease survived longer than five years compared to only $6 \%$ of those with late-stage disease [17]. Therefore, screening is essential as it can detect precancerous lesions and colorectal cancer early, so the prognosis is good [17].

\section{Socioeconomic status:}

Socioeconomic status has an association with colorectal cancer survival in several studies. Low socioeconomic status is mainly associated with poorer survival. The group with the most inferior socioeconomic status in the study of Hines and colleagues had a $24 \%$ higher risk of death than that of the most elevated socioeconomic status [18]. 


\section{Colorectal Cancer Screening Tests and studies.}

Several screening tests can be used to find polyps or colorectal cancer. The Task Force outlines the following colorectal cancer screening strategies. It is essential to know that if the test result is positive or abnormal on some screening tests (stool tests, flexible sigmoidoscopy, and CT colonography), a colonoscopy test is needed to complete the screening process [19].

\section{Stool Tests}

- The guaiac-based fecal occult blood test (gFOBT) uses the chemical guaiac to detect blood in the stool. It is done once a year. You receive a test kit from your health care provider for this test. You use a stick or brush to obtain a small amount of stool at home. You return the test kit to the doctor or a lab, where the stool samples are checked for the presence of blood [20].

- The fecal immunochemical test (FIT) uses antibodies to detect blood in the stool. It is done once a year in the same way as a gFOBT [20].

- The FIT-DNA test (also referred to as the stool DNA test) combines the FIT with a test that detects altered DNA in the stool. For this test, you collect an entire bowel movement and send it to a lab that is checked for cancer cells. It is done once every three years [20].

- Screening guidelines for persons at higher risk generally recommend additional types of testing (e.g., colonoscopy rather than, or in addition to, Fecal Occult Blood Test (FOBT), more frequent testing, and commencement of testing at an earlier age, compared with their average risk counterparts [21].

- Several randomized controlled trials have demonstrated that CRC mortality can be reduced by $15 \%$ to $33 \%$ through FOBT screening [22].

- Improved patient screening adherence could be achieved with patient and physician education. In a CRC screening pilot program evaluating 300 underserved women, $31 \%$ of patients were interested in undergoing FOBT, and 14\% requested CRC-related educational materials. This study emphasizes the importance of adequate education and the need to reach the underinsured populations who may lack access to and knowledge of CRC screening [23]. 
One study involved asymptomatic adults 50 to 69 years of age, compared onetime colonoscopy in 26,703 subjects with Fecal Immunochemical Testing (FIT) every two years in 26,599 subjects, found the rate of participation higher in the FIT group than in the colonoscopy group [24].

The experience at Kaiser-Permanente in California further illustrates it. Following failed efforts to implement screening by endoscopy, screening participation has consistently increased with the re-introduction of a non-invasive FIT test, despite the test having lower sensitivity [25].

\section{Flexible Sigmoidoscopy}

It is repeated every five years or ten years with a FIT every year [26].

The most commonly applied and efficient method in diagnosing CRC is endoscopy. It includes sigmoidoscopy and colonoscopy. These examinations allow one to localize the tumor and take biopsies of the large intestine for histological analysis. The sensitivity and specificity of sigmoidoscopy for polyps and extended CRC recognition is $92-97 \%$ [27].

\section{Colonoscopy}

This is similar to flexible sigmoidoscopy, except the doctor uses a more elongate, thin, flexible, lighted tube to check for polyps or cancer inside the rectum and the entire colon. The doctor can find and remove most polyps and some cancers during the test. Colonoscopy is used as a follow-up test if anything unusual is found during one of the other screening tests [28]. Every ten years (for people who do not have an increased risk of colorectal cancer) [28].

Obesity in women may affect CRC screening rates. A study found that colonoscopy rates were lowest among obese white women at $30.2 \%$ [29].

\section{CT Colonography}

Computed tomography (CT) colonography, also called a virtual colonoscopy, uses Xrays and computers to produce images of the entire colon, which are displayed on a computer screen for the doctor to analyze [30]. It is done every five years [30].

Several CRC screening methods aimed at early detection have been developed, and there is a substantial body of evidence supporting the benefits of CRC screening. Paradoxically, despite the clear and long-standing evidence that CRC screening reduces mortality and may reduce cancer incidence, participation rates in screening programs remain too low, at an estimated $65 \%$ in the US and ranging from $1.9 \%$ to $54 \%$ across Europe [31]. 


\section{Review Article}

Given the low participation in CRC screening programs despite the clear medical benefit, it is essential to understand the barriers to screening to develop successful alternative approaches. Numerous studies report behavioral as well as structural barriers that limit screening participation. These include factors specific to the tests themselves, such as embarrassment, fear of the procedure, or inconvenience, as well as broader factors such as lack of access to care, limited knowledge of screening, and a lack of physician recommendation [32].

In a recent study, through a questionnaire focusing on the first-degree relatives of Egyptian CRC patients, investigators found that all the surveyed relatives refused the screening tests, especially the invasive ones. According to the survey, the rationale for this was cultural and lack of knowledge about screening; they think that if they had no symptoms suggestive of colorectal cancer, there is no risk of colorectal cancer in the future. Most of them said we are healthy looking and have no symptoms, so why do I investigate? We believe that this is the most critical individual-related barrier -among others- against CRC screening [33].

A study that included 147 participants screened for CRC demonstrated the overall compliance rate for CRC screening was 33\%. For the most part, the reasons for noncompliance were unknown (79\%). A significant number of patients refused to be screened (20\%) [34].

Luis et al. studied 281 first-degree relatives of CRC patients and found that the compliance rate to colonoscopy was $38 \%$. Colonoscopy adherence was higher among female relatives, relatives of index patients under 65 years, and relatives with a higher degree of familial aggregation for colorectal cancer. They believed that the perception that colonoscopy and the preparation of the colon were uncomfortable or painful has a considerable influence on adherence. This was supported by findings that subjects prefer less awkward screening methods such as fecal occult blood tests. Other reasons reported for low commitment are lack of time to visit the doctor, low economic and educational levels, lack of interest, the perception of colorectal cancer as a minor problem, and, in the case of women, the fact that an endoscopist is a man [35].

Another study of 217 patients also concluded that patients preferred FOBT (43\%) or colonoscopy (40\%) as their primary screening method. Patients for whom accuracy was the most critical test feature chose colonoscopy (62\%) as their preferred test. In contrast, patients for whom the invasiveness of the trial was critical decided FOBT $(76 \%)[36]$.

Multiple studies have found that women are less likely than men to undergo CRC screening in both the United States and Canada. Disparities in the screening rates for CRC versus breast and cervical cancers may be partly due to a misperception by women and their physicians that CRC is a "man's disease." [37]. 
Review Article

It is clear from the results of the previous studies that offering a blood test as part of the screening menu further improves participation, as approximately 100 percent of subjects opted for a blood test. While it is clear that the ease and convenience of a blood test can improve screening participation, many additional factors will determine the impact of a new screening test. In addition to performance characteristics, these include guideline recommendations, health economic considerations, and cost to the patient, among others [38].

Finally, Colorectal cancer screening programs remain less prevalent because of the invasive nature of endoscopies, financial burden, time-consuming, anesthesia, lack of awareness, and lack of a commonly accepted method of screening by researchers, and other barriers to screening [39].

The situation is different in Egypt, and in many developing countries with no screening or surveillance for colorectal cancer, so much effort is needed to persuade populations for screening programs in such countries. Also, the orientation of the general population to this serious cancer is required. Establishing national guidelines to consider the population's culture and customs is mandatory. It is essential to teach general practitioners and young physicians about the benefits of screening and the deadly results of advanced colonic carcinoma [40].

Conclusion: Providing non-invasive screening choices can augment participation in programs for colorectal cancer screening. The success of a screening program depends not only on having tests with acceptable performance but also on the willingness of the target population to participate.

Footnotes.

Citation of this article: Mohamed SY et al. Adherence to colorectal cancer screening. African journal of gastroenterology and hepatology [Internet]. Egypt's

Presidential Specialized Council for Education and Scientific Research; 2021 October 27;4(1):44-54.

Peer- Reviewers: Hanan Soliman (professor of hepatology, gastroenterology, and infectious diseases), prof Amr Shaban (professor of internal medicine), prof Hany Sadek (internal medicine department), prof Mohamed Refaee (prof of tropical medicine, Zagazig University).

E- Editor: Prof Salem Y Mohamed, Prof Emad Fawzi Hamed (professor of internal medicine, Zagazig University).

Copyright $($ ). This open-access article is distributed under the Creative Commons Attribution License (CC BY). The use, distribution, or reproduction in other forums is permitted, provided the original author(s) and the copyright owner(s) are credited. The original publication in this journal is cited by accepted academic practice. No use, distribution, or reproduction is permitted, complying with these terms. 
Review Article

Disclaimer: All claims expressed in this article are solely those of the authors and do not necessarily represent their affiliated organizations or those of the publisher, the editors, and the reviewers. Any product evaluated in this article or claim that its manufacturer may make is not guaranteed or endorsed by the publisher.

\section{References}

1- American Cancer Society. Colorectal cancer facts \& figures 2020-2022. Published online. 2020:48.

2. Makhlouf NA, Abdel-Gawad M, Macros AM, Lashen SA, et al. Colorectal cancer in the Arab world: A systematic review. World J Gastrointest Oncol. 2021 Nov 15;13(11):1791-1798. doi: 10.4251/who. v13.i11.1791. PMID: 34853651; PMCID: PMC8603455.

3. Ibrahim AS, Khaled HM, Mikhail NN, Baraka H, Kamel H. Cancer incidence in Egypt: results of the national population-based cancer registry program. J Cancer Epidemiol. 2014; 2014:437971. doi: 10.1155/2014/437971. Epub 2014 Sep 21. PMID: 25328522; PMCID: PMC4189936.

4. Vuik FE, Nieuwenburg SA, Bardou $M$ et al. Increasing incidence of colorectal cancer in young adults in Europe over the last 25 years. Gut.2019; 68:1820-1826. 5. Chan P, Ngu J, Poh Z, Soetikno R. Colorectal cancer screening. Singapore Med J [Internet]. 2017 Jan [cited 2018 Oct 18];58(1):24-8. Available from: http://www.smj.org.sg/article/colorectal-cancer-screening.

6. Johnson CM, Wei C, Ensor JE et al. (2013). Meta-analyses of colorectal cancer risk factors. Cancer causes \& control, 24:1207\{1222\}.

7.Li J, Wang Z, Yuan et al. (2015). The prognostic significance of age in operated and non-operated colorectal cancer."BMC Cancer, 15:83.

8- Stornes T, Wibe A, Romundstad et al. (2014). Outcomes of rectal cancer treatment - influence of age? International journal of colorectal disease, 29:825\{834\}.

9. Rex, Douglas K., David A. Johnson, Joseph C. Anderson, Phillip S. Schoenfeld, Carol A. Burke, and John M. Inadomi. "American College of Gastroenterology guidelines for colorectal cancer screening 2008." Official Journal of the American College of Gastroenterology| ACG 104, no. 3 (2009): 739-750.

10.Lydrup ML and Hoglund P (2015). Gender aspects of survival after surgical treatment for rectal cancer. Colorectal Disease, 17:390\{396\}.

11. Cheung WY, Shi Q, O'connell M, Cassidy J et al. (2013). The predictive and prognostic value of sex in early-stage colon cancer: a pooled analysis of 33,345 patients from the ACCENT database. Clinical colorectal cancer, 12:179\{187\}. 12- Veach E, Xique I, Johnson J et al. (2014). Race matters: analyzing the relationship between colorectal cancer mortality rates and various factors within respective racial groups." Frontiers in public health, 2:239.

13. Sharp L, McDevitt J, Brown C et al. (2017). Smoking at diagnosis significantly decreases 5-year cancer-specific survival in a population-based cohort of 18166 colon cancer patients. Alimentary pharmacology \& therapeutics, 45:788 $\{800\}$.

14. Croft B, Reed M, Patrick C et al. (2018). Diabetes, obesity, and metabolic syndrome as prognostic factors in stages I to III colorectal cancer patients. Journal of gastrointestinal cancer, 
Review Article

15. Li J, Liu J, Gao et al. (2017). Increased mortality for colorectal cancer patients with preexisting diabetes mellitus: an updated meta-analysis. Oncotarget, 8: 62478. 16. Lee YC, Lee YL, Chuang JP et al. (2013). Differences in survival between the colon and rectal cancer from SEER data. PloS one, 8: e78709.

17. Li Q, Cai G, Li D et al. (2014). After surgery, there was a better long-term survival in young patients with non-metastatic colorectal cancer, an analysis of 69,835 patients in the SEER database. PLoS One, 9: e93756.

18- Hines R, Markossian T, Johnson A et al. (2014). Geographic residency status and census tract socioeconomic status as determinants of colorectal cancer outcomes. American journal of public health, 104: e63\{e71\}.

19.Lin JS, Perdue LA, Henrikson NB, Bean SI, Blasi PR. Screening for Colorectal Cancer: An Evidence Update for the US Preventive Services Task Force. Evidence Synthesis No. 202. Agency for Healthcare Research and Quality; 2021. AHRQ publication 20-05271-EF-1.

20. Division of Cancer Prevention and Control, Centers for Disease Control and Prevention.2021.

21. Ryan J Courtney, Christine L Paul, Mariko L Carey, et al., (2013). Daniel Barker and Jody Simmons. A population-based cross-sectional study of colorectal cancer screening practices of first-degree relatives of colorectal cancer patients. BMC Cancer, 13:13

22.Brenner H, Chang-Claude J, Seiler CM, et al., (2011). Protection from colorectal cancer after colonoscopy: a population-based case-control study. Ann Intern Med, 154:22-30.

23.Miesfeldt S, Hayden C, Apedoe $\mathrm{N}$ et al., (2010). Colorectal cancer screening pilot program for underserved women in Cumberland County, Maine. J Community Health; 35:109-114

24.Enrique Q, Antoni C, Luis B, et al. (2012). Colonoscopy versus Fecal Immunochemical Testing in Colorectal-Cancer Screening. N Engl J Med; 366:697706.

25. Moiel D, Thompson J (2011). Early detection of colon cancer-the Kaiser Permanente northwest 30-year history: how do we measure success? Is it the test, the number of tests, the stage, or the percentage of screen-detected patients? Perm J, $4: 30-38$.

26.McCarthy BD and Moskowitz MA). Screening flexible sigmoidoscopy: patient attitudes and compliance. J Gen Intern Med;8: 120 - 5.

27.Lieberman DA, Rex DK, Winawer SJ, (2012). Levin TR; United States MultiSociety Task Force on Colorectal Cancer. Guidelines for colonoscopy surveillance after screening and polypectomy: a consensus update by the US Multi-Society Task Force on Colorectal Cancer. Gastroenterology; 143: 844-57

28.McEntire J, Sahota J, Hydes T et al. (2013). An evaluation of patient attitudes to colonoscopy and the importance of endoscopist interaction and the endoscopy environment to satisfaction and value. Scandinavian Journal of Gastroenterology, 48(3), 366-373.

29. Leone LA, Campbell MK, Satia JR, et al., (2010). Race moderates the relationship between obesity and colorectal cancer screening in women. Cancer Causes Control; 21:373-385. 
Review Article

30.Sali L, Grazzini G \& Mascalchi M (2017). CT colonography: role in FOBT-based screening programs for colorectal cancer. Clinical Journal of Gastroenterology, 10(4), 312-319.

31.Kuipers EJ, Rosch T and Bretthauer M (2013). Colorectal cancer screening optimizing current strategies and new directions. Nat Rev Clin Oncol, 10(3):130-142. 32.Gimeno García AZ (2012). Factors influencing colorectal cancer screening participation. Gastroenterol Res Pract, 2012:483417.

33. Elsayed AF, Ali HS, El-Maghawry HA, Atia H, Mohamed SY. Compliance of Blood Relatives of Colorectal Cancer Patients to Colorectal Screening Guidelines. J Gastrointest Cancer. 2021 Nov 10. doi: 10.1007/s12029-021-00756-1. Epub ahead of print. PMID: 34761339.

34. Ashish M, Khalid A, and James W. (2007). Colorectal cancer screening: a retrospective study of compliance with guidelines in university-based primary care practice. Quality in Primary Care; 15:151-5.

35.Luis B, Cristina S, Leire Z et al., (2007). Low adherence to colonoscopy in the screening of first-degree relatives of patients with colorectal cancer. Gut; 56:17141718.

36. Ling BS, Moskowitz MA, Wachs D, et al., Attitudes toward colorectal cancer screening tests. J Gen Intern Med; 16:822 - 30 .

37.Lisa L. Chu, Stefanie Weinstein, and Judy Yee. (2011). Colorectal Cancer

Screening in Women: An Underutilized Lifesaver. AJR; 196:303-310.

38.Adler A, Sebastian Geiger, Anne Keil, et al. (2014). Improving compliance to colorectal cancer screening using blood and stool-based tests in patients refusing screening colonoscopy in Germany. BMC Gastroenterology.14:183.

39. Onyoh EF, Hsu WF, Chang LC et al. (2019). The rise of colorectal cancer in Asia: epidemiology, screening, and management. Current gastroenterology reports, 21(8), 36

40. Zaki MM, said MAA, Mohamed MMA, abdelrasheed MF, Adel M, Farag MM, et al. Colorectal cancer screening: a minireview. African journal of gastroenterology and hepatology [Internet]. Egypt's Presidential Specialized Council for Education and Scientific Research; 2019 Aug 6;2(1):65-73. 